\title{
Selective Utilization of $\mathrm{N}$-acetyl Groups in Chitin for Transamidation of Amines
}

\author{
Yu Xin ${ }^{1,2,3}$, Xiaojun Shen ${ }^{1,2,3}$, Huizhen Liu ${ }^{1,2,3 *}$ and Buxing Han ${ }^{1,2,3}$ \\ ${ }^{1}$ Beijing National Laboratory for Molecular Sciences, CAS Key Laboratory of Colloid and Interface and Thermodynamics, CAS \\ Research/Education Center for Excellence in Molecular Sciences, Institute of Chemistry, Chinese Academy of Sciences, Beijing, \\ China, ${ }^{2}$ School of Chemistry and Chemical Engineering, University of Chinese Academy of Sciences, Beijing, China, ${ }^{3}$ Physical \\ Science Laboratory, Huairou National Comprehensive Science Center, Beijing, China
}

\section{OPEN ACCESS}

Edited by:

Yanqin Wang,

East China University of Science and

Technology, China

Reviewed by:

$\mathrm{Hu} \mathrm{Li}$,

Guizhou University, China Heng Zhang,

Guizhou University, China

${ }^{*}$ Correspondence:

Huizhen Liu

liuhz@iccas.ac.cn

Specialty section: This article was submitted to

Catalytic Engineering,

a section of the journal

Frontiers in Chemical Engineering

Received: 29 November 2020

Accepted: 28 December 2020

Published: 29 January 2021

Citation:

Xin Y, Shen X, Liu H and Han B (2021) Selective Utilization of $\mathrm{N}$-acetyl Groups in Chitin for Transamidation of Amines.

2:634983.

doi: $10.3389 /$ fceng.2020.634983
The selective transformation of chitin into various renewable $\mathrm{N}$-containing chemicals and medicines has attracted increasing attention. However, the $\mathrm{N}$-acetyl groups in chitin construct strong hydrogen bond networks, which restricts its depolymerization and transformation. The selective conversion of robust chitin commonly requires considerable base catalysts to remove the $\mathrm{N}$-acetyl group as a byproduct in advance, which is non-compliance with the principle of atomic economy. Herein, for the first time we demonstrate a novel approach to achieve the selective utilization of the $\mathrm{N}$-acetyl group in chitin for transamidation of chitin with amines. A series of amine derivatives, mainly including aliphatic amine, cyclic amine and functionalized aromatic amine, could be selectively converted into the corresponding amide products frequently found in pharmaceuticals. Furthermore, the solid residue after removing the acetyl group (denoted as De-chitin) with the sufficient exposure of $-\mathrm{NH}_{2}$ groups as a solid base catalyst shows excellent performance in the aldol condensation reaction of furfural and acetone to produce fuel precursors. Our process provides a strategy that exploiting every functional group adequately in substrates to obtain value-added chemicals.

Keywords: chitin, $\mathrm{N}$-acetyl group, transamidation, amine, amide

\section{INTRODUCTION}

Chitin, the second most abundant biopolymer on the earth after cellulose, consists of $\mathrm{N}$-acetylglucosamine units with $\beta$-1,4-glycosidic linkages. (Yabushita et al., 2015; Yan and Chen et al., 2015). Chitin is considered as the most promising biomass sources for the production of renewable $\mathrm{N}$-containing chemicals and materials due to its high nitrogen content of around $7 \%$ by weight. (Omari et al., 2012; Chen et al., 2014; Chen et al., 2017; Kobayashi et al., 2017, 2019; Osada et al., 2019; Pham et al., 2019; Dai et al., 2020). In spite of the enormous economic and environmental interests, the existence of acetyl groups is the main challenge for the exploitation of chitin biomass, which seriously hampers its transformation into fuels and fine chemicals (Fang and Fan et al., 2019). It has been reported that deacetylation is commonly inevitable for the valorization of chitin. However, deacetylation is notorious due to the use of plenty of concentrated, corrosive basic solutions (Chen et al., 2017). Moreover, acetyl groups in chitin are removed as a byproduct, which results in low atomic utilization and is contrary to the theme of Green Chemistry.

Amides are enormously important building blocks in organic synthesis, and they serve as precursors for many value-added compounds, mainly including agrochemicals, pharmaceuticals, 
organic materials and polymers (Pattabiraman and Bode et al., 2011; Lundberg et al., 2014; Kniss, 2017). Hitherto, several synthetic measures have been achieved. Generally, the preparation of amide involves hydroamination of alkynes (Uenoyama et al., 2005; Valeur and Bradley et al., 2009), hydration of nitriles (Goto et al.,2008; Raman et al., 2009; Williams et al., 2011) and the coupling of carboxylic acids, aldehydes and alcohols with amines (Srinivas and Das, 2003; Hosseini-Sarvari and Sharghi et al., 2006; Gunanathan and Milstein, 2007, Milstein, 2011; Nordstrom et al., 2008; Arnold et al., 2008; Zweifel et al., 2009; Watson et al., 2009; Soule et al., 2011; Lundberg et al., 2012; Gosh et al., 2012; Yamaguchi et al., 2012). Additionally, transamidation is a convenient and straightforward method for exchanging the constituents of two different amide groups and some significant progress has been achieved in recent years (Eldred et al., 2003; Eldred et al., 2008; Nguyen et al., 2012, Allen et al., 2012; Zhang et al., 2012; Nageswara et al., 2014; Becerra-Figueroa et al. 2014; Garg et al. 2017; Yin et al., 2019; Ghosh et al., 2019). Chitin possesses enormous $\mathrm{N}$-acetyl groups and can be recognized as a kind of renewable amide compounds. Selective deacetylation of $\mathrm{N}$-acetyl groups with additional amine source through transamidation not only generated high-value amide products, but benefited to its further valorization. To the best of our knowledge, transamidation reaction regarding renewable chitin as amide source has never been reported.

Aldol condensation is known as an important $\mathrm{C}-\mathrm{C}$ bond forming reaction and a cornerstone of synthetic chemistry (Yutthalekha et al., 2017; Xu et al., 2017; Ngo et al., 2019). Generally, aldol reaction proceeds in the presence of acid, base or acid-base bifunctional catalysts. Among those catalysts, base catalysts gave remarkable catalytic performance owing to its strong ability to abstract $a$-protons and active substrate (Ngo et al., 2019). Homogeneous base catalysts, such as $\mathrm{NaOH}$ and $\mathrm{KOH}$, were widely employed in the aldol reaction, but showed some disadvantages, mainly involving equipment corrosion, nonrecyclability and complex separation (West et al., 2008; Fakhfakh et al., 2008; Xing et al., 2010; Ramirez-Barria et al., 2016; Gu et al., 2017). To overcome these drawbacks, heterogeneous base catalysts (MgAl-hydrotalcite and $\mathrm{MgO}$ et al.) were considered as suitable alternatives, while the challenge of catalyst stability existed in the presence of water generated in the process of aldol condensation (Yang et al., 2013; Shen et al., 2016; Bing et al., 2017; Ngo et al., 2018). Therefore, exploring a solid base catalyst with outstanding water-resistance to enhance aldol reaction is very urgent. Chitosan is the partially deacetylated form of chitin. Chitosan with considerable $-\mathrm{NH}_{2}$ groups, as a promising renewable solid base catalyst, emerged to promote the aldol condensation reaction, due to its water resistance and recyclability (Sakthivel and Dhakshinamoorthy et al., 2017; Rani et al., 2018; Meninno, 2020; Anbu et al., 2020).

We report here a novel catalytic system where the deacetylation of $\mathrm{C} 2$-acetamido groups to $\mathrm{C} 2$-amido groups selectively occurred through a transamidation process with amines. The amine sources mainly include aliphatic amine, cyclic amine and functionalized aromatic amine, generating the corresponding amide as the desired product with excellent yield. FT-IR spectra and XRD pattern demonstrated that the transamidation reaction proceeded mainly on the surface of chitin. Additionally, the solid residue after transamidation reaction (De-chitin), as a solid base catalyst with exposure of lots of $-\mathrm{NH}_{2}$ groups, exhibited outstanding catalytic activity on aldol condensation of furfural with acetone to produce fuel precursor. Our work offers a strategy to sufficiently use every functional groups in the substrate for the production of valueadded chemicals from an atomic economy perspective.

\section{EXPERIMENTAL SECTION}

\section{Chemicals and Materials}

Furfural (98\%), acetone (99.9\%), N, N-dimethylformamide (98\%), chitin and chitosan were purchased from TCI. Aniline (99\%), propylamine (99\%), butylamine (99\%), cyclopentamine (99\%), cyclohexylamine (99\%), benzylamine (99\%), p-toluidine (99\%), $p$-anisidine (99\%), p-chloroaniline (98\%), p-bromoaniline (99\%) were purchased from Aladdin. $\mathrm{NaOH}(99 \%), \mathrm{Fe}(\mathrm{OAc})_{2}(95 \%)$, $\mathrm{Co}(\mathrm{OAc})_{2}$ (98\%), $\mathrm{Ni}(\mathrm{OAc})_{2}$ (97\%), $\mathrm{Cu}(\mathrm{OAc})_{2}$ (99\%), $\mathrm{AlCl}_{3}(99 \%)$, $\mathrm{CoCl}_{2}$ (97\%), $\mathrm{CuCl}_{2}$ (98\%) were purchased from Alfa Aesar.

\section{Catalytic Reactions}

The transamidation reaction was performed in a $50 \mathrm{ml}$ flask at ambient pressure. Typically, chitin $(0.10 \mathrm{~g})$, catalyst $(0.03 \mathrm{~g})$, amine and DMF $(3 \mathrm{ml})$ were placed into the flask. The reactor was purged with $\mathrm{N}_{2}$ three times. The reaction was then performed at $140^{\circ} \mathrm{C}$ under magnetic stirring for $12 \mathrm{~h}$. The liquid solution was separated from the solid residual by centrifugation and analyzed qualitatively by GC-MS (Agilent 5977A) and quantitatively by GC equipped with a flame ionization detector (FID, Agilent 4890D) using dodecane as the internal standard. The experimental section of transamidation reaction involving other molecules is the same as the above process. Additionally, the solid residue (De-chitin) was collected, washed with ethanol and then dried in an oven for the next step.

The yield of amide product was calculated by using the equation: amide yield $=$ (the actual moles of amide) $/$ (the theoretical moles of amide). The theoretical moles of amide were obtained according to the degree of acetylation (DA) of chitin which was calculated based on elemental analysis (EA) (Chen et al., 2014).

$$
\mathrm{DA}=[(\mathrm{C} / \mathrm{N}-5.14) / 1.72] \times 100 \%
$$

$\mathrm{C} / \mathrm{N}$ means the ratio of carbon to nitrogen (w/w).

The aldol condensation of furfural with acetone was conducted in a $10 \mathrm{ml}$ Teflon-lined stainless-steel autoclave. Furfural $(0.48 \mathrm{~g}$, $5 \mathrm{mmol})$, acetone $(1.16 \mathrm{~g}, 20 \mathrm{mmol})$ and De-chitin catalyst $(0.10 \mathrm{~g})$ were transferred into the autoclave. The reactor was charged to $1 \mathrm{MPa} \mathrm{N}_{2}$ pressure. The reaction was then performed at $140^{\circ} \mathrm{C}$ under magnetic stirring for $12 \mathrm{~h}$. After the aldol condensation reaction, the autoclave was quenched in an ice-water bath to room temperature. The liquid solution was separated from the solid residual by centrifugation and analyzed qualitatively by GC-MS (Agilent, 5977A) and quantitatively by GC equipped with a flame ionization detector (FID, Agilent, 4890D) using dodecane as the 


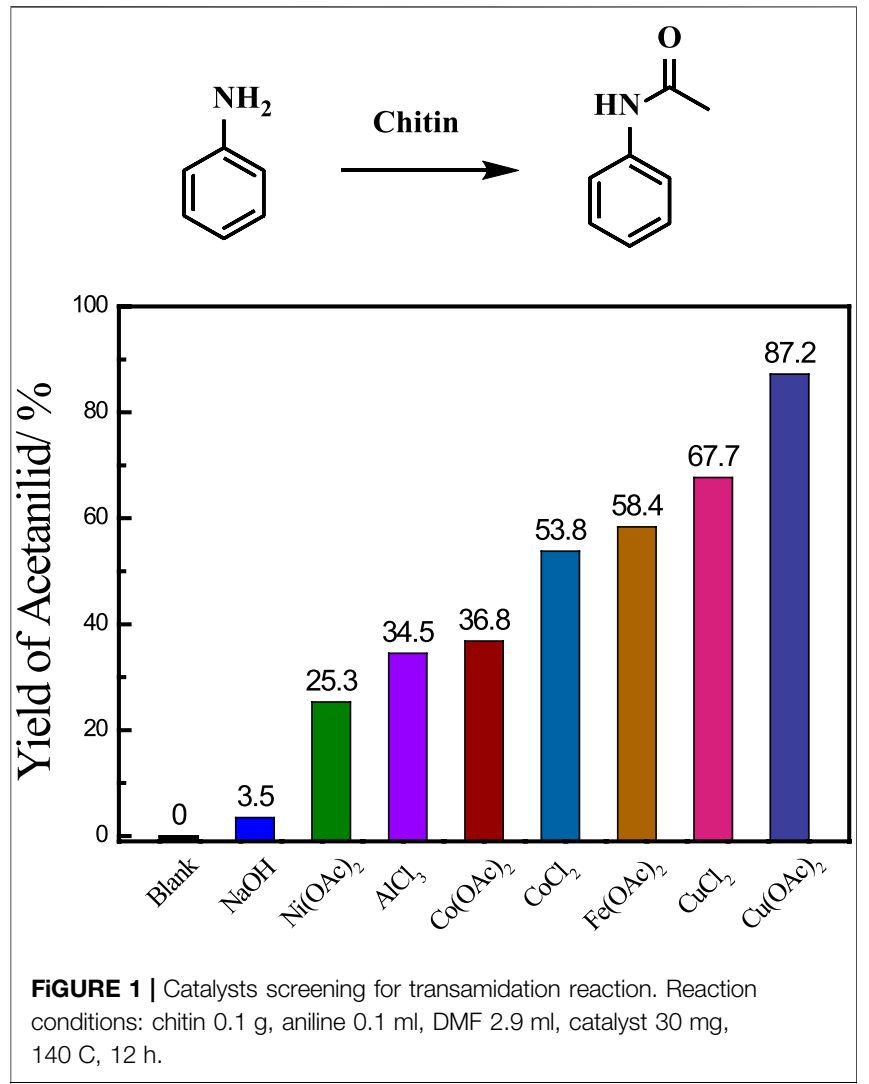

internal standard. Conversion of furfural and yield of products were calculated by using the equation:

$$
\begin{aligned}
\text { Furfural conversion }= & \begin{aligned}
(\text { moles of reacted furfural }) / \\
(\text { moles of staring furfural }) \times 100 \% .
\end{aligned} \\
\mathrm{C} 8 \text { yield }= & \begin{aligned}
(\text { moles of } \mathrm{C} 8 \text { product }) /(\text { moles of staring furfural }) \\
\times
\end{aligned} \\
\mathrm{C} 13 \text { yield }= & (\text { moles of C13 product }) / \\
& (\text { moles of staring furfural } / 2) \times 100 \%
\end{aligned}
$$

\section{Characterization}

Powder X-ray diffraction (XRD) patterns were obtained by Rigaku $\mathrm{D} / \mathrm{max}-2500 \mathrm{X}$-ray diffractometer (excitation source: $\mathrm{Cu} \mathrm{Ka}$ radiation, $\lambda=0.15406 \mathrm{~nm}$; tube voltage: $40 \mathrm{kV}$; tube current: $200 \mathrm{~mA}$ ).

FT-IR spectra of commercially available chitin, chitosan and De-chitin were recorded with a TENSOR 27 spectrometer. The samples were blended with $\mathrm{KBr}$ for IR characterization.

\section{RESULTS AND DISCUSSION}

Initially, aniline was employed as an amine source to investigate the transamidation reaction between chitin and aniline, and various catalysts were screened for the production of acetanilid at $140^{\circ} \mathrm{C}$ (Figure 1). The degree of acetylation (DA) in chitin is $95.4 \%$ based on the elemental analysis and the theoretical moles of acetanilid were calculated to $58.5 \mathrm{mg}$ when the amount of chitin substrate is $100 \mathrm{mg}$ (Supplementary Table S1) (Chen et al., 2014). The transamidation reaction did not occur without catalyst and homogeneous base catalyst $(\mathrm{NaOH})$ showed poor performance with only $3.5 \%$ yield of acetanilid as the desired product. Lewis acid catalysts are known to be effective for this reaction. We evaluated lots of Lewis acid catalysts, mainly including $\mathrm{Ni}(\mathrm{OAc})_{2}, \mathrm{AlCl}_{3}$, $\mathrm{Co}(\mathrm{OAc})_{2}, \mathrm{CoCl}_{2}, \mathrm{Fe}(\mathrm{OAc})_{2}, \mathrm{CuCl}_{2}$ and $\mathrm{Cu}(\mathrm{OAc})_{2}$, and found that all those catalysts could produce target molecular. Among them, $\mathrm{Cu}(\mathrm{OAc})_{2}$ provided the best performance with $87.2 \%$ yield of acetanilid after $12 \mathrm{~h}$ at $140^{\circ} \mathrm{C}$. Those results obviously indicated that metal core played a significant role in the transamidation reaction and the excellent catalytic activity of $\mathrm{Cu}$ catalyst may be attributed to the proper combination between acetyl groups in chitin and it.

With this promising result, the factors of reaction were further optimized, including solvents, reaction time, reaction temperature and the amount of $\mathrm{Cu}$ catalyst. The use of solvents involving tetrahydrofuran, acetonitrile, $p$-xylene, isopropanol and cyclohexane, did not provide the desired product, which demonstrates the critical role of DMF in promoting transamidation reaction (Figure 2A). Figure 2B illustrated the influence of reaction time on the transamidation reaction and the yield of acetanilide increased before $12 \mathrm{~h}$. Prolonging the reaction time, the yield of the target product showed little change. In addition, the influence of reaction temperature on the yield of acetanilid was also investigated (Figure 2C) and the results showed that the reaction temperature played an important role in the transamidation reaction. The most suitable reaction temperature is $140^{\circ} \mathrm{C}$ and decreasing the reaction temperature proved to be unfavorable for the transamidation reaction. Moreover, the effect of the amounts of $\mathrm{Cu}$ catalyst on the yield of acetanilid was also studied. The yield of the target product increased continuously with the increasing amount of $\mathrm{Cu}(\mathrm{OAc})_{2}$ at the beginning and reached a plateau when the amount of $\mathrm{Cu}(\mathrm{OAc})_{2}$ was above $30 \mathrm{mg}$, revealing that $\mathrm{Cu}(\mathrm{OAc})_{2}$ catalyzed the reaction during the reaction process (Figure 2D). A moderate yield of $55.3 \%$ was still achieved when the amount of $\mathrm{Cu}$ catalyst was decreased to $15 \mathrm{mg}$.

Based on the previous literature report (Ma and Gong, 2018) and our results, a reasonable reaction mechanism has been proposed (Scheme 1). Initially, the carbonyl group of chitin is activated by $\mathrm{Cu}(\mathrm{OAc})_{2}$ catalyst through coordination. The activated chitin then undergoes nucleophilic attack by amine, which results in the formation of tetrahedral intermediate 1) With a proton transfer, the sterically congested intermediate 1 breaks down to generate intermediate 2) Finally, the target molecular, corresponding amide, is produced to finish the catalytic cycle.

Infrared spectroscopy is an important tool to acquire information on surface functional groups and all samples were analyzed by FT-IR (Supplementary Figure S1). The bands ranging from 2,800 to $3,500 \mathrm{~cm}^{-1}$ are assigned to the vibrations of $-\mathrm{CH},-\mathrm{NH}$ and $-\mathrm{OH}$ groups, and the peaks between 1500 and $1670 \mathrm{~cm}^{-1}$ represent amide bands. Commercial available chitosan and chitin gave remarkably different FT-IR spectra from $1,000-4,000 \mathrm{~cm}^{-1}$. Three obvious peaks at $1,115,1,550$, and $3,250 \mathrm{~cm}^{-1}$ in the chitin spectrum are absent in that of chitosan. Most importantly, the FT-IR spectrum 

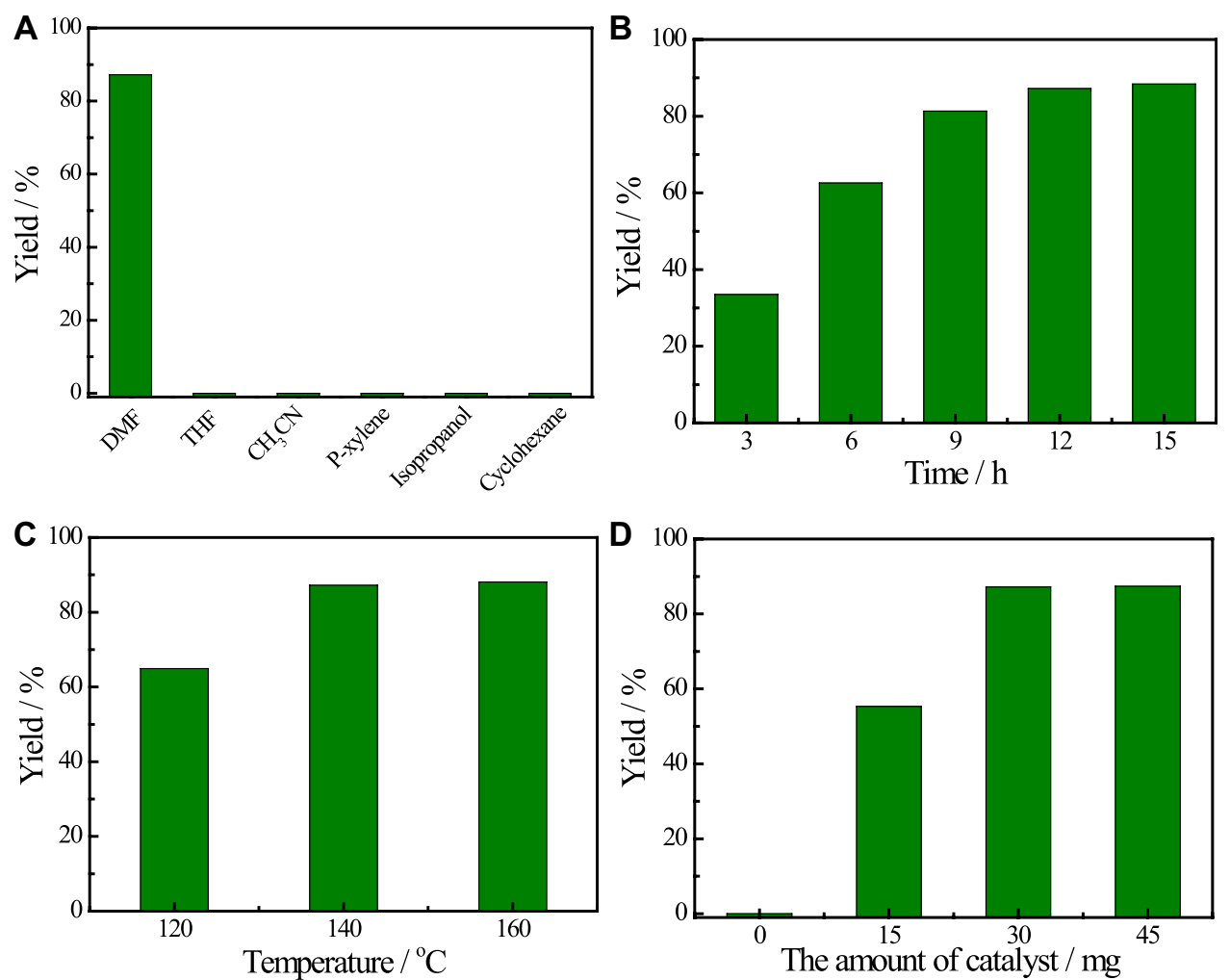

FIGURE 2 | The optimization of reaction factors. (A) solvents screening. (B) reaction time. (C) reaction temperature. (D) the amount of catalyst. Reaction conditions: chitin $0.1 \mathrm{~g}$, aniline $0.1 \mathrm{ml}$, DMF $2.9 \mathrm{ml}$, catalyst $30 \mathrm{mg}, 140^{\circ} \mathrm{C}, 12 \mathrm{~h}$.

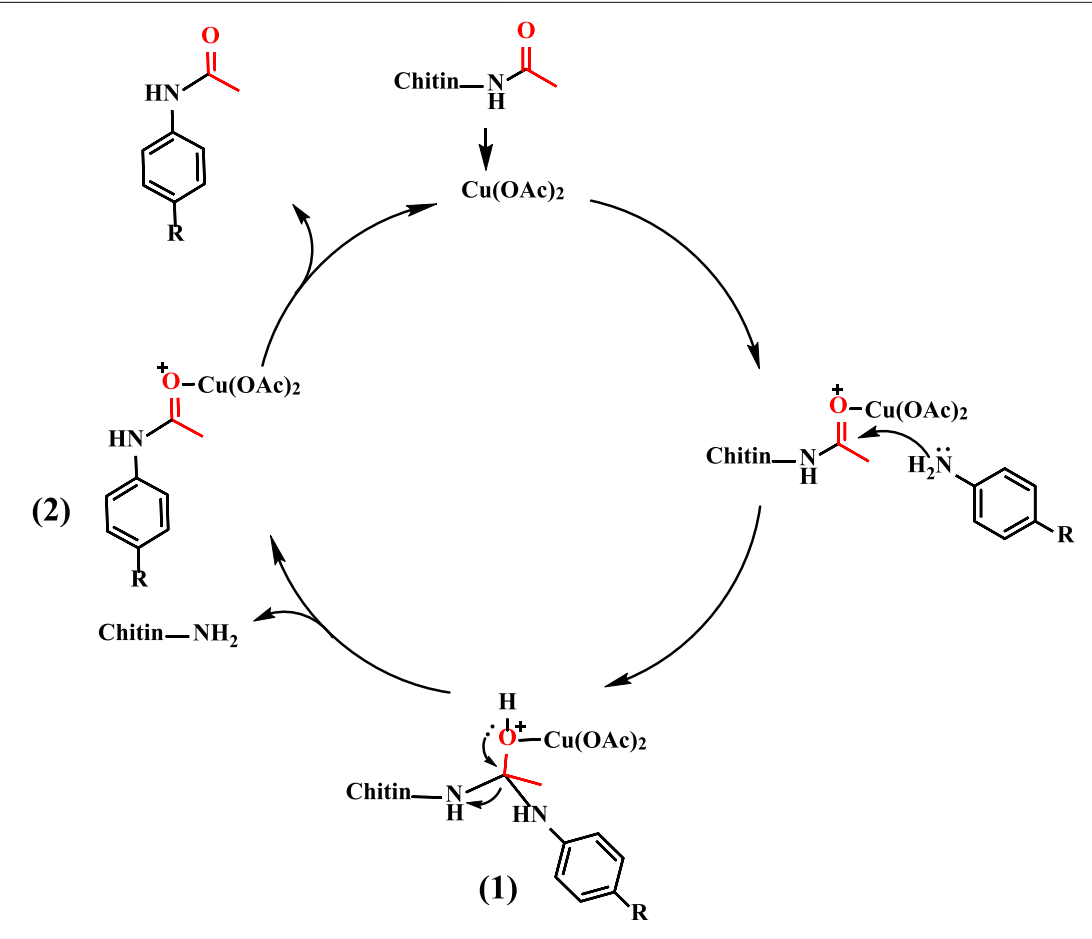

SCHEME 1 | Possible reaction mechanism. 
TABLE 1 | Substrate scope in terms of amines.

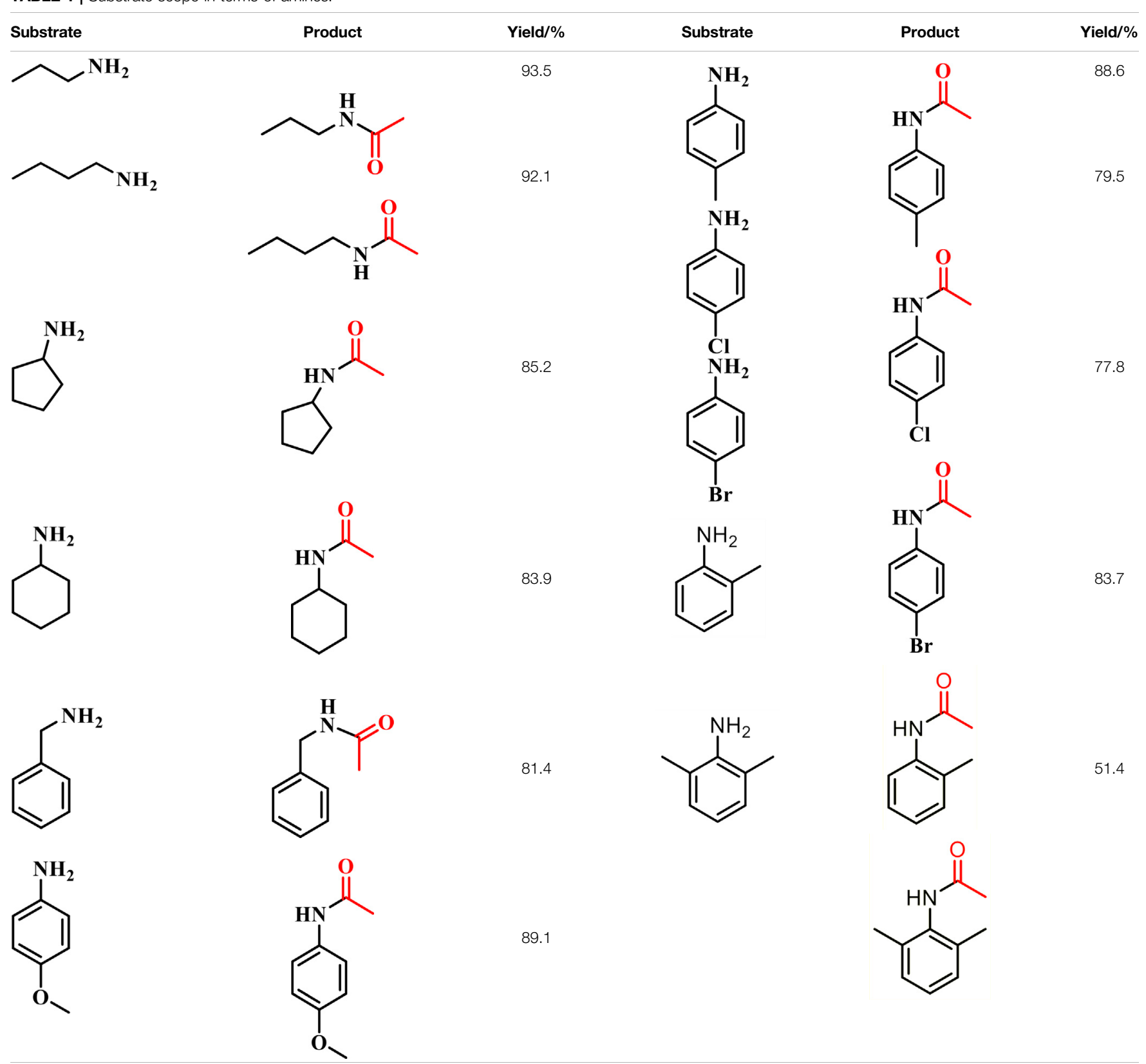

Reaction conditions: chitin $0.1 \mathrm{~g}$, amine $0.1 \mathrm{ml}$, DMF $2.9 \mathrm{ml}$, catalyst $30 \mathrm{mg}, 140^{\circ} \mathrm{C}, 12 \mathrm{~h}$.

of De-chitin highly resembles that of chitosan, indicating effective deacetylation of $\mathrm{N}$-acetyl groups in the catalytic system.

The XRD patterns of the three samples are depicted in Supplementary Figure S2 to investigate their bulk phase structure. Chitin exhibits characteristic diffraction peaks centered at 9.6 and 19.6 of diffraction angle $2 \theta$, representing the (020) and (110) planes of crystalline chitin, respectively. While chitosan has no obvious diffraction peaks, indicating its amorphous structure. Notably, the XRD pattern of De-chitin is similar with that of commercially available chitin. Namely, the original chitin structure is maintained during the $\mathrm{Cu}(\mathrm{OAc})_{2^{-}}$ catalyzed process. FT-IR and XRD characterizations indicated the deacetylation of $\mathrm{C} 2$-acetamido groups to $\mathrm{C} 2$-amido groups selectively occurred on the surface of chitin.

With the optimized conditions in hand, the amine scope of the $\mathrm{Cu}(\mathrm{OAc})_{2}$-catalyzed transamidation reaction was examined (Table 1). The results revealed broad applicability of this reaction to various amines, mainly including aliphatic amines, cyclic amines and functionalized aromatic amines, generating the corresponding amide products. Particularly noteworthy is that aliphatic amine, such as propylamine and butylamine, can undergo transamidation with chitin even without catalyst and the yields of corresponding amides are up to 93.5 and $92.1 \%$, respectively. The promising result is mostly ascribed to the 

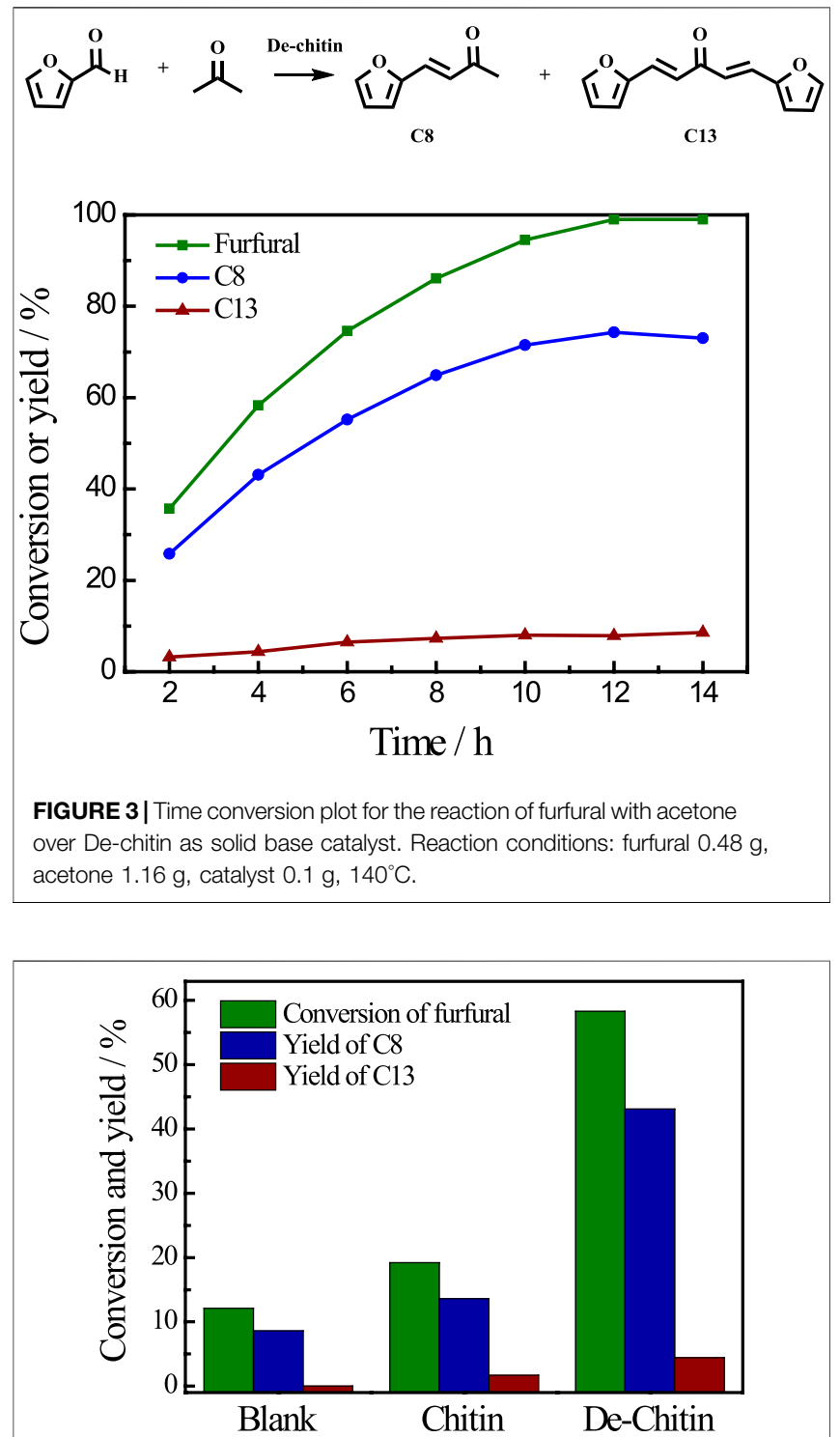

FIGURE 4 | Aldol reaction catalyzed by various catalysts. Reaction conditions: furfural $0.48 \mathrm{~g}$, acetone $1.16 \mathrm{~g}$, catalyst $0.1 \mathrm{~g}, 140^{\circ} \mathrm{C}, 4 \mathrm{~h}$.

stronger nucleophilic capability of aliphatic amine than others. When cyclopentamine and cyclohexylamine were employed as amine sources, excellent yields of corresponding amide products were achieved (85.2 and 83.9\%, respectively). For functionalized aromatic amines, different functional groups on the benzene ring were compatible with the reaction, including methyl, methoxy, bromide and chlorine groups. The reaction proceeded well with $p$-methyl or $p$-methoxy aniline as substrate. However, the chlorine and bromine groups were found to have a negative influence on the transamidation reaction, giving the corresponding amines 79.5 and $77.8 \%$ yield, respectively. Furthermore, to investigate the effect of steric hindrance on the reaction, $o$-toluidine and 2,6-dimethyaniline were employed to conduct the transamidation reaction. Notably, the yield of corresponding amide decreased to 83.7 and $51.4 \%$ with

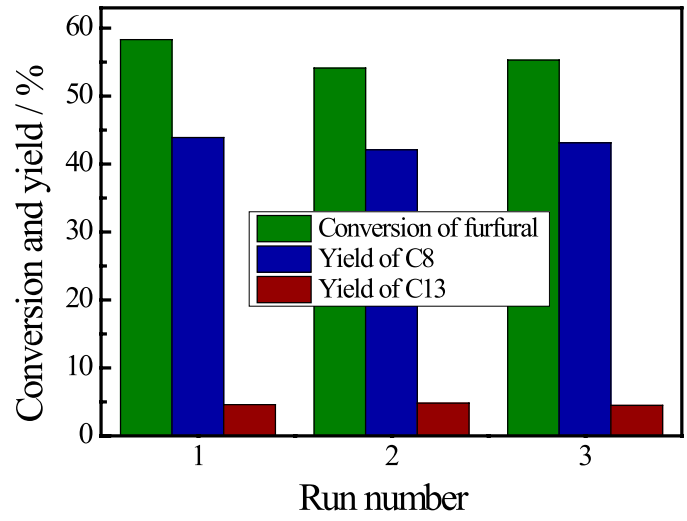

FIGURE 5 | Recyclability test of De-chitin for aldol condensation. Reaction conditions: furfural $0.48 \mathrm{~g}$, acetone $1.16 \mathrm{~g}$, De-chitin $0.1 \mathrm{~g}$, $140^{\circ} \mathrm{C}, 4 \mathrm{~h}$.

$o$-toluidine and 2,6-dimethyaniline as amine source as compared to that yield $(87.2 \%)$ with aniline as amine source. The result demonstrated that the reaction was influenced by the steric effect of substrate, apart from nucleophilicity of the amine source.

After reaction, the solid residue (denoted as De-chitin) was separated by centrifugation, washed with ethanol and dried in an oven for the next step utilization. The initial $\mathrm{Cu}$ content is $9.54 \%$ based on the weight of chitin in catalytic system. We tested the $\mathrm{Cu}$ content in solid residue after reaction and found that only $0.07 \%$ $\mathrm{Cu}$ metal exist in the deacetylated chitin after centrifugation (Supplementary Table S2). The aldol condensation of furfural with acetone was employed as a model reaction to evaluate the catalytic performance of De-chitin. Figure 3 illustrated the influence of reaction time on the aldol reaction. The conversion of furfural increased continuously with the prolongation of reaction time in the beginning and reached a plateau at $12 \mathrm{~h}$. De-chitin catalyst showed remarkable catalytic performance and furfural was completely converted over De-chitin catalyst after $12 \mathrm{~h}$ at $140^{\circ} \mathrm{C}$, with $74.3 \% \mathrm{C} 8$ yield and $7.9 \% \mathrm{C} 13$ yield. Figure 4 revealed that the conversion of furfural was $12.1 \%$ even without catalyst under solvent-free condition. However, the conversion of furfural and yields of condensed products just increased slightly over chitin catalyst, mainly due to the existence of $\mathrm{C} 2$-acetamido groups and inadequate exposure of $-\mathrm{NH}_{2}$ groups. Notably, De-chitin catalyst exhibited excellent catalytic performance for aldol condensation reaction, with the furfural conversion up to $58.3 \%$, which is much higher than that with chitin as catalyst. The deacetylation of $\mathrm{C} 2$-acetamido groups to $\mathrm{C} 2$-amido groups, namely the adequate exposure of surface $-\mathrm{NH}_{2}$ groups in the De-chitin sample, is responsible for the enhanced reaction activity.

The recyclability of the De-chitin catalyst for aldol condensation of furfural with acetone was investigated by reusing the catalyst in consecutive catalytic run. The catalyst was separated by simple filtration, washed with ethanol and oven dried for five hours. It can be seen from Figure 5 that no obvious change was observed in the catalytic activity and product selectivity even after three runs. The recyclability test reveals 
the potential application of De-chitin as a heterogeneous catalyst for aldol condensation.

\section{CONCLUSION}

In summary, $\mathrm{N}$-acetyl groups in chitin were removed with additional amine sources through transamidation to generate corresponding amide products. The amine sources mainly involved aliphatic amines, cyclic amines and functionalized aromatic amines and the yields of corresponding amides commonly exceed $75 \%$. Notably, aliphatic amines, such as propylamine and butylamine, can undergo transamidation with chitin even without catalyst and the yields of corresponding amides are up to 93.5 and $92.1 \%$, respectively. The promising result is mostly ascribed to the stronger nucleophilic capability of aliphatic amine than others. Additionally, FT-IR and XRD results demonstrated that deacetylation reaction only occurred on the surface of chitin. Furthermore, the solid residue after transamidation reaction (Dechitin) was applied as a heterogeneous base catalyst for aldol condensation of furfural with acetone under mild reaction conditions and showed excellent catalytic activity. The catalyst was recovered and reused for three runs without any drop in its activity and selectivity. This work provides a measure to sufficiently use functional groups in the substrate to produce high-value chemicals from an atomic economy perspective.

\section{REFERENCES}

Al-Mourabit, C. L., Atkinson, B. N., and Williams, J. M. (2012). Transamidation of primary amides with amines using hydroxylamine hydrochloride as an inorganic catalyst. Angew Chem. Int. Ed. Engl. 51, 1383-1386. doi:10.1002/ anie. 201107348

Allen, C. L., and Williams, J. M. (2011). Metal-catalysed approaches to amide bond formation. Chem. Soc. Rev. 40, 3405-3415. doi:10.1039/c0cs00196a

Anbu, N., Maheswari, R., Elamathi, V., Varalakshmi, P., and Dhakshinamoorthy, A. (2020). Chitosan as a biodegradable heterogeneous catalyst for Knoevenagel condensation between benzaldehydes and cyanoacetamide. Catal. Commun. 138, 105954. doi:10.1016/j.catcom.2020.105954

Arnold, K., Davies, B., Hérault, D., and Whiting, A. (2008). Asymmetric direct amide synthesis by kinetic amine resolution: a chiral bifunctional aminoboronic acid catalyzed reaction between a racemic amine and an achiral carboxylic acid. Angew Chem. Int. Ed. Engl. 47, 2673-2676. doi:10. 1002/anie. 200705643

Becerra-Figueroa, L., Ojeda-Porras, A., and Gamba-Sánchez, D. (2014). Transamidation of carboxamides catalyzed by $\mathrm{Fe}(\mathrm{III})$ and water. J. Org. Chem. 79, 4544-4552. doi:10.1021/jo500562w

Bing, W., Zheng, L., He, S., Rao, D., Xu, M., Zheng, L., et al. (2017). Insights on active sites of CaAl-hydrotalcite as a high-performance solid base catalyst toward aldol condensation. ACS Catal. 8, 656-664. doi:10.1021/acscatal. $7 \mathrm{~b} 03022$

Chen, X., Chew, S. L., Kerton, F. M., and Yan, N. (2014). Direct conversion of chitin into a N-containing furan derivative. Green Chem. 16, 2204-2212. doi:10.1039/ c3gc42436g

Chen, X., Yang, H., Zhong, Z., and Yan, N. (2017). Base-catalysed, one-step mechanochemical conversion of chitin and shrimp shells into low molecular weight chitosan. Green Chem. 19, 2783-2792. doi:10.1039/c7gc00089h

Dai, J., Li, F., and Fu, X. (2020). Towards shell biorefinery: advances in chemicalcatalytic conversion of chitin biomass to organonitrogen chemicals. ChemSusChem. 13, 6498-6508. doi:10.1002/cssc.202001955

\section{DATA AVAILABILITY STATEMENT}

The original contributions presented in the study are included in the article/Supplementary Material, further inquiries can be directed to the corresponding author.

\section{AUTHOR CONTRIBUTIONS}

$\mathrm{YX}, \mathrm{HL}$, and $\mathrm{BH}$ conceived the projects. YX carried out the experiments, analyzed data in the assist of XS and wrote the manuscript in the guidance of $\mathrm{HL}$ and $\mathrm{BH}$.

\section{FUNDING}

The work was supported financially by National Natural Science Foundation of China (21871277) and Beijing Municipal Science \& Technology Commission (Z191100007219009).

\section{SUPPLEMENTARY MATERIAL}

The Supplementary Material for this article can be found online at: https://www.frontiersin.org/articles/10.3389/fceng.2020.634983/ full\#supplementary-material.

Dander, J. E., Baker, E. L., and Garg, N. K. (2017). Nickel-catalyzed transamidation of aliphatic amide derivatives. Chem. Sci. 8, 6433-6438. doi:10.1039/c7sc01980g

Eldred, S. E., Stone, D. A., Gellman, S. H., and Stahl, S. S., 2003). Catalytic transamidation under moderate conditions. J. Am. Chem. Soc. 125, 3422-3423. doi:10.1021/ja028242h

Fakhfakh, N., Cognet, P., Cabassud, M., Lucchese, Y., and De Los Ríos, M. D. (2008). Stoichio-kinetic modeling and optimization of chemical synthesis: application to the aldolic condensation of furfural on acetone. Chem. Eng. Process 47, 349-362. doi:10.1016/j.cep.2007.01.015

Ghosh, S. C., Ngiam, J. S., Seayad, A. M., Tuan, D. T., Chai, C. L., and Chen, A. (2012). Copper-catalyzed oxidative amidation of aldehydes with amine salts: synthesis of primary, secondary, and tertiary amides. J. Org. Chem. 77, 8007-8015. doi:10.1021/jo301252c

Ghosh, T., Jana, S., and Dash, J. (2019). KOtBu-promoted transition-metal-free transamidation of primary and tertiary amides with amines. Org. Lett. 21, 6690-6694. doi:10.1021/acs.orglett.9b02306

Goto, A., Endo, K., and Saito, S. (2008). Rh (I)-catalyzed hydration of organonitriles under ambient conditions. Angew Chem. Int. Ed. Engl. 47, 3607-3609. doi:10.1002/anie.200800366

Gu, M., Xia, Q., Liu, X., Guo, Y., and Wang, Y., 2017). Synthesis of renewable lubricant alkanes from biomass-derived platform chemicals. ChemSusChem. 10, 4102-4108. doi:10.1002/cssc.201701200

Gunanathan, C., Ben-David, Y., and David, M., 2007). Direct synthesis of amides from alcohols and amines with liberation of $\mathrm{H}_{2}$. Science 317, 790-792. doi:10. $1126 /$ science. 1145295

Hosseini-Sarvari, M., and Sharghi, H. (2006). $\mathrm{ZnO}$ as a new catalyst for $\mathrm{N}$-formylation of amines under solvent-free conditions. J. Org. Chem. 71, 6652-6654. doi:10.1021/jo060847z

Hülsey, M. J., Yang, H., and Yan, N. (2018). Sustainable routes for the synthesis of renewable heteroatom-containing chemicals. ACS Sustain. Chem. Eng. 6, 5694-5707. doi:10.1021/acssuschemeng.8b00612

Jing, Y., Guo, Y., Xia, Q., Liu, X., and Wang, Y. (2019a). Catalytic production of value-added chemicals and liquid fuels from lignocellulosic biomass. Inside Chem. 5, 2520-2546. doi:10.1016/j.chempr.2019.05.022 
Jing, Y., Xin, Y., Guo, Y., Liu, X., and Wang, Y. (2019b). Highly efficient Nb2O5 catalyst for aldol condensation of biomass-derived carbonyl molecules to fuel precursors. Chin. J. Catal. 40, 1168-1177. doi:10.1016/S1872-2067(19)63371-1

Kniss, A. R. (2017). Long-term trends in the intensity and relative toxicity of herbicide use. Nat. Commun. 8, 14865. doi:10.1038/ncomms14865

Kobayashi, H., Techikawara, K., and Fukuoka, A. (2017). Hydrolytic hydrogenation of chitin to amino sugar alcohol. Green Chem. 19, 3350-3356. doi:10.1039/c7gc01063j

Lundberg, H., Tinnis, F., and Adolfsson, H. (2012). Direct amide coupling of nonactivated carboxylic acids and amines catalysed by zirconium(IV) chloride. Chemistry 18, 3822-3826. doi:10.1002/chem.201104055

Lundberg, H., Tinnis, F., Selander, N., and Adolfsson, H. (2014). Catalytic amide formation from non-activated carboxylic acids and amines. Chem. Soc. Rev. 43, 2714-2742. doi:10.1039/c3cs60345h

Ma, J., and Gong, H. (2018). Cobalt(II)-catalyzed N-acylation of amines through a transamidation reaction. Eur. J. Org Chem., 2018, 4940-4948. doi:10.1002/ejoc. 201800253

Meninno, S. (2020). Valorization of waste: sustainable organocatalysts from renewable resources. ChemSusChem. 13, 439-468. doi:10.1002/cssc.201902500

Milstein, L. U., Vogt, H., and Madsen, R. (2008). Amide synthesis from alcohols and amines by the extrusion of dihydrogen. J. Am. Chem. Soc. 130, 17672-17673. doi:10.1021/ja808129p

Nageswara, R. S., Chandra, M. D., and Adimurthy, S. (2014). Chitosan: an efficient recyclable catalyst for transamidation of carboxamides with amines under neat conditions. Green Chem. 16, 4122-4126. doi:10.1039/c4gc01402b

Ngo, D. T., Sooknoi, T., and Resasco, D. E. (2018). Improving stability of cyclopentanone aldol condensation MgO-based catalysts by surface hydrophobization with organosilanes. Appl. Catal. B Environ. 237, 835-843. doi:10.1016/j.apcatb.2018.06.044

Ngo, D. T., Tan, Q., Wang, B., and Resasco, D. E. (2019). Aldol condensation of cyclopentanone on hydrophobized MgO. promotional role of water and changes in the rate-limiting step upon organosilane functionalization. ACS Catal. 9, 2831-2841. doi:10.1021/acscatal.8b05103

Omari, K. W., Dodot, L., and Kerton, F. M. (2012). A simple one-pot dehydration process to convert $\mathrm{N}$-acetyl-D-glucosamine into a nitrogen-containing compound, 3acetamido-5-acetylfuran. ChemSusChem. 5, 1767-1772. doi:10.1002/cssc.201200113

Osada, M., Shoji, S., Suenaga, S., and Ogata, M. (2019). Conversion of N-acetyl-dglucosamine to nitrogen-containing chemicals in high-temperature water. Fuel Process. Technol. 195, 106154. doi:10.1016/j.fuproc.2019.106154

Pattabiraman, V. R., and Bode, J. W. (2011). Rethinking amide bond synthesis. Nature 480, 471-479. doi:10.1038/nature10702

Pham, T. T., Lindsay, A. C., Chen, X., Gözaydin, G., Yan, N., and Sperry, J. (2019). Transferring the biorenewable nitrogen present in chitin to several $\mathrm{N}$-functional groups. Sustainable Chemistry and Pharmacy 13, 100143. doi:10.1016/j.scp.2019.100143

Ramirez-Barria, C., Guerrero-Ruiz, A., Castillejos-López, E., Rodríguez-Ramos, I., Durand, J., Volkman, J., et al. (2016). Surface properties of amphiphilic carbon nanotubes and study of their applicability as basic catalysts. RSC Adv. 6, 54293-54298. doi:10.1039/c6ra08032d

Ramón, R. S., Marion, N., and Nolan, S. P. (2009). Gold activation of nitriles: catalytic hydration to amides. Chemistry 15, 8695-8697. doi:10.1002/chem.200901231

Rani, D., Singla, P., and Agarwal, J. (2018). 'Chitosan in water' as an eco-friendly and efficient catalytic system for Knoevenagel condensation reaction. Carbohydr. Polym. 202, 355-364. doi:10.1016/j.carbpol.2018.09.008

Sagawa, T., Kobayashi, H., Murata, C., Shichibu, Y., Konishi, K., and Fukuoka, A. (2019). Catalytic conversion of a chitin-derived sugar alcohol to an amidecontaining isosorbide analog. ACS Sustain. Chem. Eng. 7, 14883-14888. doi:10. 1021/acssuschemeng.9b02985

Sakthivel, B., and Dhakshinamoorthy, A. (2017). Chitosan as a reusable solid base catalyst for knoevenagel condensation reaction. J. Colloid Interface Sci. 485, 75-80. doi:10.1016/j.jcis.2016.09.020

Sheng, X., Li, N., Li, G., Wang, W., Wang, A., Cong, Y., et al. (2016). Direct synthesis of gasoline and diesel range branched alkanes with acetone from lignocellulose. Green Chem. 18, 3707-3711. doi:10.1039/c6gc01127f

Soulé, J. F., Miyamura, H., and Kobayashi, S. (2011). Powerful amide synthesis from alcohols and amines under aerobic conditions catalyzed by gold or gold/ iron, -nickel or -cobalt nanoparticles. J. Am. Chem. Soc. 133, 18550-18553. doi:10.1021/ja2080086
Srinivas, K. V. N. S., and Das, B. (2003). A highly convenient, efficient, and selective process for preparation of esters and amides from carboxylic acids using $\mathrm{Fe}(3+)$ K-10 montmorillonite clay. J. Org. Chem. 68, 1165-1167. doi:10.1021/jo0204202

Hoerter, J. M., Otte, K. M., Gellman, S. H., Cui, Q., and Stahl, S. S., (2008). Discovery and mechanistic study of $\mathrm{Al}(\mathrm{III})$-catalyzed transamidation of tertiary amides. J. Am. Chem. Soc. 130, 647-654. doi:10.1021/ja0762994

Nguyen, T. B., Sorres, J., Tran, M. Q., Ermolenko, L., and Al-Mourabit, A., (2012). Boric acid: a highly efficient catalyst for transamidation of carboxamides with amines. Org. Lett. 14, 3202-3205. doi:10.1021/ol301308c

Uenoyama, Y., Fukuyama, T., Nobuta, O., Matsubara, H., and Ryu, I. (2005). Alkyne carbonylation by radicals: tin-radical-catalyzed synthesis of alphamethylene amides from 1-alkynes, carbon monoxide, and amines. Angew Chem. Int. Ed. Engl. 44, 1075-1078. doi:10.1002/anie.200461954

Valeur, E., and Bradley, M. (2009). Amide bond formation: beyond the myth of coupling reagents. Chem. Soc. Rev. 38, 606-631. doi:10.1039/b701677h

Wang, J., Li, N., Li, G., Wang, W., Wang, A., Wang, X., et al. (2013). Solvent-free synthesis of $\mathrm{C} 10$ and $\mathrm{C} 11$ branched alkanes from furfural and methyl isobutyl ketone. ChemSusChem. 6, 1149-1152. doi:10.1002/cssc.201300318

Watson, A. J., Maxwell, A. C., and Williams, J. M. (2009). Ruthenium-catalyzed oxidation of alcohols into amides. Org. Lett. 11, 2667-2670. doi:10.1021/ ol $900723 \mathrm{v}$

West, R. M., Liu, Z. Y., Peter, M., Gärtner, C. A., and Dumesic, J. A. (2008). Carboncarbon bond formation for biomass-derived furfurals and ketones by aldol condensation in a biphasic system. J. Mol. Catal. Chem. 296, 18-27. doi:10. 1016/j.molcata.2008.09.001

Xing, R., Subrahmanyam, A. V., Olcay, H., Qi, W., Pendse, H., and Huber, G. W. (2010). Production of jet and diesel fuel range alkanes from waste hemicellulose-derived aqueous solutions. Green Chem. 12, 1933-1946. doi:10.1039/c0gc00263a

Xu, J., Li, N., Yang, X., Li, G., Wang, A., Cong, Y., et al. (2017). Synthesis of diesel and jet fuel range alkanes with furfural and angelica lactone. ACS Catal. 7, 5880-5886. doi:10.1021/acscatal.7b01992

Yabushita, M., Kobayashi, H., Kuroki, K., Ito, S., and Fukuoka, A. (2015). Catalytic depolymerization of chitin with retention of $\mathrm{N}$-acetyl group. ChemSusChem. 8, 3760-3763. doi:10.1002/cssc.201501224

Yamaguchi, K., Kobayashi, H., Oishi, T., and Mizuno, N. (2012). Heterogeneously catalyzed synthesis of primary amides directly from primary alcohols and aqueous ammonia. Angew Chem. Int. Ed. Engl. 51, 544-547. doi:10.1002/anie.201107110

Yan, N., and Chen, X. (2015). Sustainability: don't waste seafood waste. Nature 524, 155-157. doi:10.1038/524155a

Ye, W., Ma, H., Liu, L., Yu, J., Lai, J., Fang, Y., et al. (2019). Biocatalyzed route for the preparation of surface-deacetylated chitin nanofibers. Green Chem. 21, 3143-3151. doi:10.1039/c9gc00857h

Yin, J., Zhang, J., Cai, C., Deng, G. J., and Gong, H. (2019). Catalyst-free transamidation of aromatic amines with formamide derivatives and tertiary amides with aliphatic amines. Org. Lett. 21, 387-392. doi:10.1021/acs.orglett.8b03542

Yutthalekha, T., Suttipat, D., Salakhum, S., Thivasasith, A., Nokbin, S., Limtrakul, J., et al. (2017). Aldol condensation of biomass-derived platform molecules over amine-grafted hierarchical FAU-type zeolite nanosheets (Zeolean) featuring basic sites. Chem Commun (Camb) 53, 12185-12188. doi:10.1039/c7cc06375j

Zhang, M., Imm, S., Bähn, S., Neubert, L., Neumann, H., and Beller, M. (2012). Efficient copper(II)-catalyzed transamidation of non-activated primary carboxamides and ureas with amines. Angew Chem. Int. Ed. Engl. 51, 3905-3909. doi:10.1002/anie.201108599

Zweifel, T., Naubron, J. V., and Grützmacher, H. (2009). Catalyzed dehydrogenative coupling of primary alcohols with water, methanol, or amines. Angew Chem. Int. Ed. Engl. 48, 559-563. doi:10.1002/anie.200804757

Conflict of Interest: The authors declare that the research was conducted in the absence of any commercial or financial relationships that could be construed as a potential conflict of interest.

Copyright () 2021 Xin, Shen, Liu and Han. This is an open-access article distributed under the terms of the Creative Commons Attribution License (CC BY). The use, distribution or reproduction in other forums is permitted, provided the original author(s) and the copyright owner(s) are credited and that the original publication in this journal is cited, in accordance with accepted academic practice. No use, distribution or reproduction is permitted which does not comply with these terms. 